\title{
A inclusão de ações da América Latina sob o ponto de vista do investidor brasileiro: inferências sobre os pesos na fronteira eficiente
}

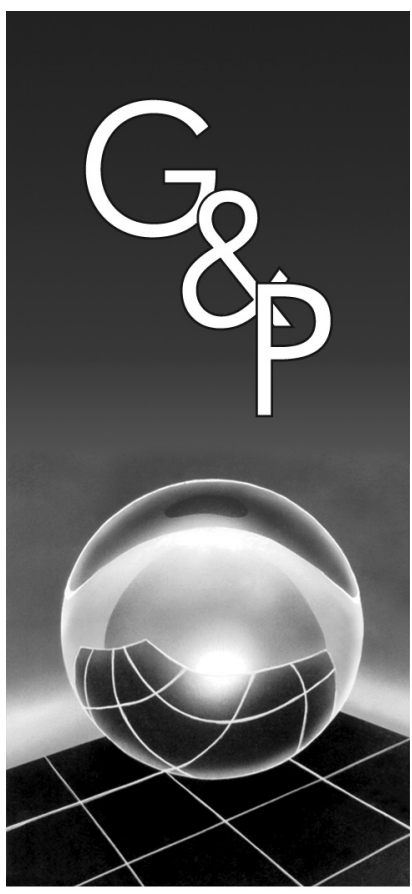

Marco Antonio Cunha de Oliveira

Lílian Simone Aguiar da Silva

\section{Resumo}

O problema de como alocar recursos de forma eficiente tem sido uma das questões fundamentais em Finanças. Se os fatores domésticos tendem a fazer com que os ativos num determinado mercado se movimentem em conjunto, os investidores procuram diversificar o risco nacional pela aplicação em outros mercados. Este tema tem sido tipicamente analisado no contexto retorno-risco, entretanto, um dos maiores problemas é por não reconhecer a incerteza nos parâmetros de entrada, dando origem ao risco de estimação. Este trabalho analisa se a alocação em ações de outros países da América Latina permite melhorar a fronteira eficiente sob o ponto de vista do investidor brasileiro. É utilizada a combinação de inferências estatísticas propostas por Britten-Jones (1999) para portfólios de tangência e Kempf e Memmel (2006) para o portfólio de risco mínimo global. Os resultados permitiram verificar que a adição do investimento em ações de outros países latinos melhoraria a fronteira eficiente sob o ponto de vista do investidor local, com pesos estatisticamente significantes.

Palavras-chave: Carteiras. Diversificação. Inferência.

\section{Introdução}

O problema de como alocar recursos de forma eficiente tem sido uma das questões fundamentais em Finanças. Em seu artigo clássico, Markowitz (1952) propõe o processo de seleção de carteiras a partir dos parâmetros retorno esperado versus risco, estabelecendo a fronteira eficiente como o conjunto dos portfólios em que para um dado risco é obtido o maior retorno possível, ou, para um dado retorno o menor risco possível, risco este mensurado como desvio-padrão dos retornos.

Entretanto, um dos maiores problemas da otimização média versus desvio-padrão tradicional é não reconhecer a incerteza nos parâmetros de entrada (JORION, 1992). Este problema tem sido tratado sob o tema risco de estimação.

Uma vez que fatores nacionais tendem a ser dominantes, os investidores procuram diversificar seu risco doméstico (SOLNIK, 1996). Assim, seria possível melhorar a relação retorno-risco pela inclusão de outros mercados. Solnik (1974) já abordava este efeito de diversificação internacional, que, de acordo com Jorion (1992), tradicionalmente tem sido analisado no contexto retorno-risco. É importante ressaltar que outros aspectos podem ser analisados quando se questiona a inclusão de ações de outro mercado à carteira doméstica, tais como: diferenças culturais; legais; de liquidez (SOLNIK, 1996).
Com o desenvolvimento de novos testes no âmbito da teoria de carteiras, este tema de diversificação internacional tem sido novamente explorado em trabalhos recentes, tendo sua atenção voltada para a inferência estatística sobre os resultados obtidos. Neste contexto, este artigo analisa o efeito de diversificação e sua validade estatística sob o ponto de vista do investidor brasileiro, utilizando a combinação das metodologias de inferência por regressão propostas por Britten-Jones (1999), Kempf e Memmel (2006).

$\mathrm{O}$ artigo será desenvolvido nas seguintes etapas: o item dois apresenta a base teórica da determinação da fronteira eficiente e testes recentes sobre diversificação internacional; o item três detalha a metodologia a ser implementada e a base de dados; o item quatro desenvolve o trabalho proposto sob o ponto de vista do investidor brasileiro; e o último item comenta as evidências encontradas e apresenta sugestões para trabalhos posteriores.

\section{A determinação da fronteira eficiente, risco de estimação e a inferência sobre os pesos na carteira}

A seleção de carteiras a partir do critério de dois parâmetros retorno esperado versus risco pode ser justificada 
pela distribuição dos retornos dos ativos segundo uma distribuição normal ou pela caracterização de uma função de utilidade quadrática por parte do investidor. Entretanto, Kroll, Levy e Markowitz (1984) mostram que a utilização do critério de dois parâmetros apresenta uma boa aproximação à solução pela maximização direta da utilidade esperada mesmo para diferentes funções de utilidade e alguma rejeição de normalidade por parte do retorno dos ativos.

Dado um universo de $\mathrm{n}$ ativos representados por seu vetor de retornos esperados $\mu$ e pela matriz de covariâncias $\Sigma$ entre os retornos dos ativos, então uma carteira formada com vetor de pesos $\omega$ terá como parâmetros (Equação 1 e 2):

Retorno esperado da carteira:

$$
\mu_{P}=\omega^{\prime} \mu
$$

Variância da carteira:

$$
\sigma_{P}^{2}=\omega^{\prime} \Sigma \omega
$$

em que: $\mu=(n, 1) ; \Sigma=(n, n) ; \omega=(n, 1)$ são as devidas dimensões.

A partir destes parâmetros os portfólios na fronteira eficiente podem ser obtidos pela seguinte formulação:

$$
\begin{gathered}
\text { Minimize } \sigma_{P}^{2}=\omega^{\prime} \Sigma \omega \\
\operatorname{Sob} / \mu_{P}=\omega^{\prime} \mu \\
\omega^{\prime} l=1
\end{gathered}
$$

em que 1 é um vetor unitário coerente em dimensão.

Desta forma, os pesos para o portfólio de risco mínimo global G são dados por (CAMPBELL; LO e MACKINLAY, 1997) (Equação 3):

$$
\omega_{G}=\left(\Sigma^{-1} \mathfrak{l}\right) /\left(\imath^{\prime} \Sigma^{-1} \mathfrak{l}\right)
$$

Se existe a possibilidade de tomar e aplicar recursos a uma taxa livre de risco $r_{F}$ (Figura 1), a solução para o portfólio de risco $\mathrm{T}$ tangente à fronteira eficiente que passa por $r_{F}$ é determinada pelos seguintes pesos (CAMPBELL; LO e MACKINLAY, 1997) (Equação 4):

$$
\omega_{T}=\left(\Sigma^{-1}\left(\mu-r_{F} \imath\right)\right) /\left(\imath^{\prime} \Sigma^{-1}\left(\mu-r_{F} \imath\right)\right)
$$

Neste caso, todas as carteiras eficientes serão determinadas por combinações entre a taxa livre de risco e o portfólio eficiente T.

Contudo, de acordo com Jorion (1992), um dos maiores problemas da otimização média versus desvio-padrão tradicional é não reconhecer a incerteza nos parâmetros de entrada, problema que tem sido tratado sob o tema risco de estimação. Kempf e Memmel (2006) definem risco de estimação como: "The additional out-of-sample return variance due to errors in the estimated portfolio weights."

Em face destes erros na estimação dos parâmetros de entrada, Jorion (1992) propõe um processo de simulação para aproximar a distribuição dos pesos dos ativos que solucionam a otimização da fronteira eficiente.

Chopra e Ziemba (1993) empregam a maximização da utilidade esperada com base em uma função exponencial negativa, de forma que o problema é equivalente à análise média-variância, e verificam que em relação aos parâmetros de entrada, erros nas médias têm mais influência que erros nas variâncias e covariâncias.

Ainda, face à incerteza nos parâmetros de entrada, Michaud (1989) argumenta que os otimizadores de médiavariância são maximizadores de erro de estimação e, em Michaud (1998), utiliza simulação para estimar a fronteira eficiente a partir de portfólios equivalentes.

Com o objetivo de analisar se a inclusão de ativos melhora uma fronteira eficiente prévia em termos estatísticos, Huberman e Kandel (1987) propõem os testes de interception e spanning. Supondo um conjunto de $\mathrm{K}$ ativos básicos, se a fronteira eficiente destes $\mathrm{K}$ ativos e a dos $\mathrm{N}$ ativos adicionais têm um ponto em comum, existe interseção. $\mathrm{Se}$ a fronteira dos $\mathrm{K}$ e a dos $(\mathrm{N}+\mathrm{K})$ ativos coincidem, então há spanning, não havendo neste caso melhoria pela inclusão dos $\mathrm{N}$ ativos adicionais.

Huberman e Kandel (1987) demonstram que as hipóteses de interception e spanning podem ser testadas a partir da regressão dos retornos (r) dos $\mathrm{N}$ ativos adicionais sobre os retornos (R) dos $\mathrm{K}$ ativos originais (Equação 5).

$$
r=a+B R+\xi
$$

Sob a hipótese nula de interception existe uma constante $\varpi_{0}$ tal que (Equação 6):

$$
a=\varpi_{0}\left(\mathrm{l}_{N}-B \mathrm{1}_{K}\right)
$$

E sob a hipótese nula de spanning (Equação 7):

$$
a=0 \mathrm{e}\left(\mathrm{i}_{N}-B \mathrm{i}_{K}\right)=0
$$

De forma a tratar o problema de erro de estimação e fazer inferência sobre os pesos que solucionam a fronteira eficiente, Britten-Jones (1999) demonstra que a solução para o portfólio de tangência T equivale à solução da regressão sem intercepto de um vetor unitário sobre os retornos em excesso dos ativos. Ainda, o autor argumenta que as estatísticas tradicionais da regressão (testes t e F) podem ser utilizadas para fazer inferência sobre os pesos dos ativos na

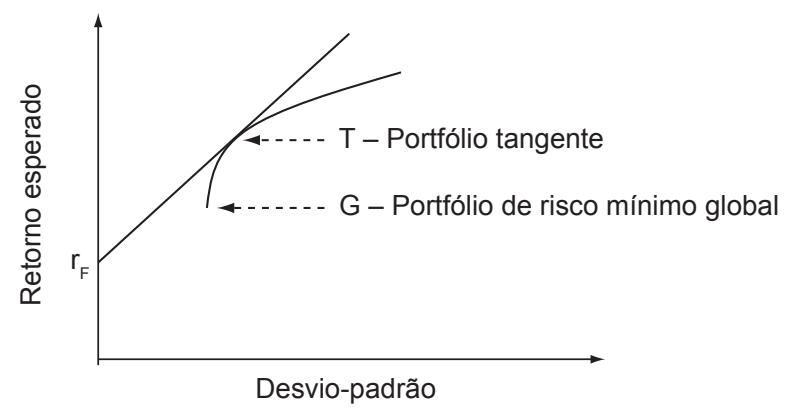

Figura 1. Portfólio G de risco mínimo global e portfólio T determinado pela tangente à fronteira eficiente para uma taxa livre de risco $r_{F}$. 
fronteira eficiente, e que estas estatísticas são exatas para amostras finitas, sob normalidade multivariada.

Por sua vez, Kempf e Memmel (2006) demonstram que o portfólio de risco mínimo global $\mathrm{G}$ pode ser obtido por uma regressão dos retornos de um dos ativos tomado como variável dependente, considerando a diferença de retornos deste para os outros ativos como variáveis independentes. Da mesma forma, Kempf e Memmel argumentam que as estatísticas usuais (testes t e F) da regressão se aplicam para fazer inferência sobre os pesos dos ativos.

Estes dois processos de inferência por regressão (BRITTEN-JONES, 1999; KEMPF e MEMMEL, 2006) serão detalhados na metodologia, sendo a base para o desenvolvimento dos objetivos do artigo.

\subsection{Aplicações recentes de fronteira eficiente ao problema de diversificação internacional}

O problema de como diversificar a carteira de investimentos local pela inclusão de outros mercados tem sido um tema tratado em Finanças há longo tempo, vide por exemplo Solnik (1974).

Dentre trabalhos mais recentes, Bruni, Fuentes e Famá (1998) analisam o efeito de inclusão de investimentos em mercados emergentes em relação a carteiras compostas por Estados Unidos, Europa e Japão. Famá e Pereira (2003) verificam com mercados latinos e Estados Unidos o aumento da correlação no tempo, entretanto, ainda comprovam ganhos de diversificação.

Britten-Jones (1999) propõe a metodologia de regressão para portfólios de tangência, que foi utilizada em seu trabalho para verificar o efeito de investimento em outros mercados sob o ponto de vista do investidor americano, sem restrição à venda a descoberto.

Kan e Zhou (2001) utilizam os testes de interception e spanning para verificar, sob o ponto de vista do investidor americano, a inclusão de sete outros mercados no portfólio.

DeRoon, Nijman e Werker (2001) estendem os testes de interception e spanning de Huberman e Kandel (1987) para o caso com restrição à venda a descoberto e custos de transação. Estes testes são então utilizados para verificar a inclusão de mercados emergentes (América Latina, Ásia, e outros) a um conjunto de ativos básicos (Estados Unidos, Europa e Japão).

Li, Sarkar e Wang (2003) implementam inferência bayesiana para verificar o efeito de diversificação sob o ponto de vista do investidor americano, com e sem restrição à venda a descoberto. É incluído na análise um universo de 15 países pertencentes ao G7 e mercados emergentes (América Latina e Ásia). Os autores argumentam que somente a partir do trabalho de Britten-Jones (1999) foi desenvolvida a teoria de distribuição amostral para o caso de portfólios sem restrição. A utilização de inferência bayesiana em diversificação internacional é também empregada por Fletcher e Marshall (2005) e, recentemente, Kempf e Memmel (2006) propõem a metodologia de regressão do portfólio de risco mínimo global para fazer inferência sobre a adição do investimento em outros países do G7 sob o ponto de vista do investidor alemão.

\section{Metodologia para inferência sobre os pesos na fronteira eficiente}

Como desenvolvido por Britten-Jones (1999), a solução do portfólio tangente à fronteira eficiente, a partir de uma determinada taxa livre de risco, equivale a uma regressão sem intercepto de um vetor unitário sobre os retornos em excesso dos ativos considerados (Equação 8).

$$
\imath=b r+\xi
$$

em que: $\imath$ é um vetor unitário coerente em dimensão;

- r são os retornos dos ativos em excesso da taxa livre de risco; e

- b são os coeficientes da regressão, sem intercepto.

Assim, os pesos $\omega$ dos ativos na carteira de tangência são obtidos a partir dos coeficientes $\hat{b}$, normalizados de forma que sua soma seja igual a 1 (Equação 9).

$$
\omega=\hat{b} /(\mathrm{l}, \hat{b})
$$

Pode-se então utilizar a estatística de teste F para verificar hipóteses sobre os pesos dos ativos, sendo esta estatística exata em amostras finitas sob a hipótese de normalidade multivariada (Equação 10).

$$
F=\left(\left(S S R_{R}-S S R_{U}\right) / q\right) /\left(S S R_{U} /(T-K)\right)
$$

Com $(q)$ e $(T-K)$ graus de liberdade em que:

- $S S R_{U}$ é a soma dos quadrados dos resíduos da regressão não restrita;

- $S S R_{R}$ é a soma dos quadrados dos resíduos da regressão restrita;

- $q$ é o número de restrições lineares;

- $K$ é o número de ativos; e

- T é o número de observações.

Para implementar a regressão com restrição é necessária a construção de uma matriz auxiliar $R$ de restrições de forma que $R^{*} b=0$, na medida em que os pesos nos ativos não são obtidos diretamente na regressão (BRITTEN-JONES, 1999).

Por sua vez, Kempf e Memmel (2006) demonstram que o portfólio de risco mínimo global $\mathrm{G}$ pode ser obtido pela seguinte regressão com intercepto (Equação 11):

$$
r_{N}=a+b\left(r_{N}-r_{I}\right)+\xi
$$

em que:

- $r_{N}$ são os retornos do ativo tomado como variável dependente;

- $r_{\mathrm{I}}$ são os retornos dos demais ativos, $I=1 . . . N-1$; 
Nesta regressão os pesos $\omega_{I}(I=1 \ldots N-1)$ são obtidos a partir dos coeficientes $\hat{b}$, e o peso do ativo $N$ é dado por 1 -soma $(\hat{b})$.

Kempf e Memmel (2006) pressupõem uma distribuição normal multivariada, embora abordem o caso de distribuições elípticas. Também, na obtenção da carteira de risco mínimo global por regressão, a estatística $\mathrm{F}$ é utilizada para fazer inferência sobre o peso dos ativos na sua composição.

Kan e Zhou (2001) argumentam que o teste de spanning corresponde a verificar se o portfólio de tangência apresenta peso zero nos ativos adicionais, o mesmo acontecendo para o portfólio de risco mínimo global, uma vez que se dois portfólios distintos da fronteira sem restrições têm peso zero nos ativos adicionais, todos os portfólios da fronteira eficiente terão. Os autores propõem uma análise em duas etapas, permitindo verificar qual a razão de rejeição da hipótese nula de spanning.

Neste sentido, a proposta de análise conjunta das metodologias de Britten-Jones (1999) e Kempf e Memmel (2006) pode ser interpretada como um teste de spanning. Contudo, uma das vantagens de sua utilização é que outras hipóteses podem ser testadas sobre os pesos dos ativos na fronteira eficiente. Por exemplo, Britten-Jones mostra que o teste de tangência pode ser utilizado para verificar se o índice de mercado é eficiente, já Kempf e Memmel testam a eficiência de um portfólio de pesos iguais.

A hipótese da normalidade multivariada, necessária aos testes, pode ser verificada com o teste proposto por Doornik e Hansen (1994) (Equação 12).

$$
Z_{1}^{\prime} Z_{1}+Z_{2}^{\prime} Z_{2} \sim \chi^{2}(2 p)
$$

em que: $Z_{1}$ e $Z_{2}$ são obtidos a partir de transformações da assimetria e curtose das séries, e $p$ é o número de ativos.

Cabe ressaltar que o teste de Doornik e Hansen se aplica a pequenas amostras e está disponível no pacote normwn.test de Wickham (2008) para o sistema $R$ (R DEVELOPMENT CORE TEAM, 2008).

Para verificar se os retornos são independentes e identicamente distribuídos (iid), será empregado o teste não paramétrico de Matilla-Garcia (2007). O teste consiste em determinar os ordenamentos em padrões na série a partir do parâmetro $\mathrm{m}$ (embedding dimension), de tal forma que são possíveis fatorial(m) padrões. Sob a hipótese nula de iid, a frequência esperada de observações em cada padrão deve ser 1/fatorial $(m)$ e a estatística de teste $X m$ tem distribuição assintótica $\chi^{2}(m !-1)$ (Equação 13).

$$
X m=\sum_{i=1}^{m !}\left(f_{i}-e_{i}\right)^{2} / e_{i}
$$

em que $f_{i}$ e $e_{i}$ são as frequências observadas e esperadas no teste.

Para a análise da inclusão de outros países da América Latina ao portfólio do investidor brasileiro serão considerados os índices de mercados de ações daqueles países, com retornos convertidos em Reais, sendo, portanto, sob o ponto de vista do investidor local. Os índices empregados são os seguintes: IBrX - Brasil; MERVAL - Argentina; IPSA - Chile; IPC - México; Lima General - Peru. Os dados de retornos para o período de 40 meses de outubro/2003 a janeiro/2007 foram calculados com base nos fechamentos mensais dos índices de cada país. Para a taxa livre de risco serão utilizados os retornos da caderneta de poupança para o mesmo período. Os dados foram obtidos a partir dos endereços www.bovespa.com.br; www.econstats.com; e www.bacen.gov.br. Todo o processo é implementado em Excel, Matlab5.3e, e no sistema $R$.

No sistema $R$ serão utilizados os pacotes normwn.test de Wickham (2008), tseries de Trapletti e Hornik (2008), e lmtest de Zeileis e Hothorn (2002).

\section{Acrescentando outros países da América Latina à carteira brasileira}

Examinando a matriz de correlações entre os retornos dos países utilizados na análise (Tabela 1) observa-se que potencialmente a inclusão de investimentos em outros mercados da América Latina pode diversificar o investimento no Brasil, face à baixa correlação com outros índices. Para verificar se este efeito existe e é estatisticamente significante, serão implementados os testes abordados na metodologia.

A primeira etapa da implementação consiste em analisar as características dos retornos dos índices selecionados e se atendem à premissa de normalidade multivariada. A partir dos resultados expostos na Tabela 2, podemos verificar que nenhum dos índices individualmente rejeita hipótese nula de normalidade dos retornos, bem como não há rejeição da hipótese nula de iid no teste de Matilla-Garcia (2007). Em resultado não reportado, foi feito o teste de KolmogorovSmirnov não rejeitando a normalidade dos retornos para o período analisado.

Quando todos os países são incluídos na análise, não é rejeitada a hipótese nula de normalidade multivariada ao nível de significância de 5\% (Tabela 3), sob o teste de Doornik e Hansen (1994). Portanto o teste F exato pode ser empregado para fazer inferência sobre os pesos nos portfólios da fronteira eficiente.

Tabela 1. Matriz de correlações entre os retornos dos países incluídos na análise.

\begin{tabular}{lccccc}
\hline & $\begin{array}{c}\text { Brasil } \\
\text { IBrX }\end{array}$ & $\begin{array}{c}\text { Argentina } \\
\text { Merval }\end{array}$ & $\begin{array}{c}\text { Chile } \\
\text { IPSA }\end{array}$ & $\begin{array}{c}\text { México } \\
\text { IPC }\end{array}$ & $\begin{array}{c}\text { Peru } \\
\text { Lima } \\
\text { General }\end{array}$ \\
\hline IBrX & 1 & & & & \\
Merval & 0,526 & 1 & & & \\
IPSA & 0,323 & 0,313 & 1 & & \\
IPC & 0,431 & 0,494 & 0,342 & 1 & \\
Lima General & 0,319 & 0,362 & 0,148 & 0,352 & 1 \\
\hline
\end{tabular}


Ao investir em um mercado externo, o investidor, ao mesmo tempo que assume uma exposição ao mercado de risco em questão, pode também assumir uma exposição à variação cambial. Neste caso, seu retorno final em sua moeda será equacionado por (ELTON et al., 2004) (Equação 14):

$$
\left(1+r_{\text {FINAL }}\right)=\left(1+r_{\text {LOCAL }}\right) *\left(1+r_{\text {CAMBIAL }}\right)
$$

em que: $r_{F I N A L}$ é o retorno final para o investidor;

- $r_{L O C A L}$ é o retorno na moeda local do investimento;

- $r_{\text {CAMBIAL }}$ é a variação da moeda local do investimento em relação à moeda do investidor.

Cabe observar que, no período em análise, todas as moedas apresentaram desvalorização em relação ao Real (Tabela 4), reduzindo a atratividade daqueles mercados para o investidor brasileiro que não tivesse feito o hedge da exposição cambial. Este trabalho não analisa o caso do investidor com hedge cambial.

Para construção da fronteira eficiente sem restrições podem ser utilizados diretamente os processos sugeridos por Britten-Jones (1999), Kempf e Memmel (2006), explicitados a seguir. Assim, são obtidos os seguintes portfólios (Tabela 5) e a fronteira eficiente equivalente (Figura 2).

Tabela 2. Parâmetros estatísticos dos retornos dos países incluídos na análise.

\begin{tabular}{lccccc}
\hline & $\begin{array}{c}\text { Brasil } \\
\text { IBrX }\end{array}$ & $\begin{array}{c}\text { Argentina } \\
\text { Merval }\end{array}$ & $\begin{array}{c}\text { Chile } \\
\text { IPSA }\end{array}$ & $\begin{array}{c}\text { México } \\
\text { IPC }\end{array}$ & $\begin{array}{c}\text { Peru } \\
\text { Lima } \\
\text { General }\end{array}$ \\
\hline Média & 3,256 & 1,618 & 1,564 & 2,484 & 4,532 \\
Desvio-padrão & 6,392 & 7,483 & 4,454 & 4,719 & 6,349 \\
Assimetria & $-0,073$ & 0,172 & $-0,311$ & $-0,205$ & $-0,102$ \\
Curtose & 3,219 & 1,981 & 2,807 & 2,416 & 2,399 \\
Jarque-Bera & 0,115 & 1,930 & 0,708 & 0,849 & 0,672 \\
p. valor $(\mathrm{JB})$ & 0,944 & 0,381 & 0,702 & 0,654 & 0,715 \\
X & 5,579 & 1,789 & 6,211 & 1,789 & 3,053 \\
p. valor $\left(\mathrm{X}_{3}\right)$ & 0,349 & 0,877 & 0,286 & 0,877 & 0,692 \\
\hline
\end{tabular}

em que: Jarque-Bera é o teste de normalidade dos retornos (GUJARATI, 2000) sob a hipótese nula de normalidade, e p.valor (JB) é a significância observada do teste. $\mathrm{X}_{3}$ é a estatística de teste de Matilla-Garcia para $\mathrm{m}=3$ sob hipótese nula de iid, e p.valor $\left(\mathrm{X}_{3}\right)$ é a significância observada do teste.

Tabela 3. Teste de normalidade multivariada de Doornik e Hansen (DH).

$\begin{array}{cr}\mathrm{DH} & 2,912 \\ \text { p. valor } & 0,983\end{array}$

Tabela 4. Variação cambial em relação ao real.

\begin{tabular}{lcrrr}
\hline & Argentina & \multicolumn{1}{c}{ Chile } & México & \multicolumn{1}{c}{ Peru } \\
\hline No período & $-0,318$ & $-0,116$ & $-0,274$ & $-0,206$ \\
Média mensal & $-0,009$ & $-0,003$ & $-0,008$ & $-0,005$ \\
Desvio-padrão & 0,030 & 0,033 & 0,030 & 0,032 \\
\hline
\end{tabular}

\subsection{Inferência sobre os pesos no portfólio de risco mínimo global}

De acordo com o processo sugerido por Kempf e Memmel (2006) é executada a regressão (11) tomando-se por variável dependente os retornos do Brasil (IBrX), cujos resultados são apresentados na Tabela 6 (Equação 15).

$$
\begin{aligned}
r_{\mathrm{t}, \text { IBRX }} & =a+b_{I}\left(r_{t, I B R X}-r_{t, \text { Merval }}\right)+\ldots+ \\
& +b_{4}\left(r_{t, \text { IBRX }}-r_{t, \text { Lima G. }}\right)+\xi
\end{aligned}
$$

A partir dos coeficientes da regressão, o peso em Brasil seria de 1 -soma $(\hat{b})=0.075$.

Para verificar se estatisticamente os pesos nos demais países são diferentes de zero, é executado o teste $\mathrm{F}$ sob a hipótese nula: $H_{0}: b_{1}=b_{2}=\ldots=b_{4}=0$.

A estatística F resultante de 19,566 e p-valor de 0,000 rejeitam a hipótese nula. Ou seja, pelo menos um dos países adicionados ao investimento em Brasil tem peso estatisticamente diferente de zero no portfólio de risco mínimo global.

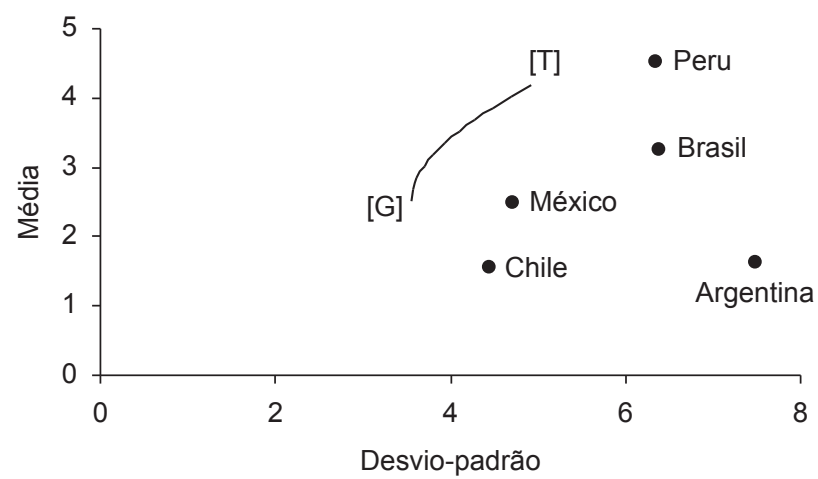

Figura 2. Segmento da fronteira eficiente sem restrição à venda a descoberto para os países considerados.

Tabela 5. Pesos dos países nos portfólios ótimos.

\begin{tabular}{lcc}
\hline & Risco mínimo (G) & $\begin{array}{c}\text { Tangente }(\mathbf{T}) \\
\text { RF = poupança }\end{array}$ \\
\hline Brasil (IBrX) & 0,075 & 0,333 \\
Argentina (Merval) & $-0,053$ & $-0,334$ \\
Chile (IPSA) & 0,473 & 0,085 \\
México (IPC) & 0,328 & 0,313 \\
Peru & & \\
(Lima General) & 0,177 & 0,604 \\
\hline
\end{tabular}

Tabela 6. Coeficientes da regressão (15) para o portfólio de risco mínimo global.

\begin{tabular}{cccrc}
\hline & Coeficientes & Erro-padrão & Estat t & P. valor \\
\hline Interseção & 2,514 & 0,669 & 3,757 & 0,001 \\
$\mathrm{~b}_{1}$ & $-0,053$ & 0,102 & $-0,516$ & 0,609 \\
$\mathrm{~b}_{2}$ & 0,473 & 0,124 & 3,817 & 0,001 \\
$\mathrm{~b}_{3}$ & 0,328 & 0,146 & 2,252 & 0,031 \\
$\mathrm{~b}_{4}$ & 0,177 & 0,100 & 1,762 & 0,087 \\
\hline
\end{tabular}




\subsection{Inferência sobre os pesos no portfólio de tangência}

Quando não existe restrição à venda a descoberto o portfólio de tangência é obtido regredindo-se o vetor unitário sobre o retorno em excesso dos ativos para todos os países, cujo resultado é apresentado na Tabela 7 (Equação 16).

$$
1=b_{1}\left(r_{t, \text { IBRX }}-r_{F}\right)+\ldots+b_{5}\left(r_{t, \text { Lima G. }}-r_{F}\right)+\xi
$$

Para verificar se estatisticamente os pesos nos demais países são diferentes de zero é executado o teste $\mathrm{F}$ sob a hipótese nula: $H_{0}: b_{2}=b_{3}=\ldots=b_{5}=0$.

A estatística $\mathrm{F}$ resultante de 2,585 e p-valor de 0,054 não rejeitam a hipótese nula. Ou seja, para o portfólio de tangência, tomado a partir da caderneta de poupança como taxa livre de risco, os pesos nos demais países não são diferentes de zero ao nível de 5\% de significância.

\subsection{Inferências para uma estratégia de diversificação proporcional ao produto interno bruto de cada país}

Uma vantagem da combinação das metodologias, como proposta, é que outras hipóteses poderiam ser testadas.

Uma estratégia simples de alocação poderia ser adotada como proporcional ao produto interno bruto de cada país (Tabela 8). Desta forma, os testes serão novamente implementados para verificar se esta carteira é eficiente para o período em análise.

Para verificar se o portfólio de risco mínimo é estatisticamente diferente da carteira proporcional ao PIB é

Tabela 7. Coeficientes da regressão (16) para o portfólio de tangência.

\begin{tabular}{cccccc}
\hline & Coeficientes & Erro-padrão & Estat t & P. valor & Pesos \\
\hline $\mathrm{b}_{1}$ & 0,032 & 0,026 & 1,226 & 0,228 & 0,333 \\
$\mathrm{~b}_{2}$ & $-0,032$ & 0,023 & $-1,397$ & 0,171 & $-0,334$ \\
$\mathrm{~b}_{3}$ & 0,008 & 0,034 & 0,241 & 0,811 & 0,085 \\
$\mathrm{~b}_{4}$ & 0,030 & 0,035 & 0,847 & 0,403 & 0,313 \\
$\mathrm{~b}_{5}$ & 0,058 & 0,022 & 2,644 & 0,012 & 0,604 \\
\hline
\end{tabular}

em que: os pesos são normalizados na última coluna de forma que somem 1, conforme equação (9).

Tabela 8. PIB de cada país e alocação proporcional.

\begin{tabular}{lcc}
\hline \multicolumn{1}{c}{ País } & PIB (US\$ milhões) & \% PIB Total \\
\hline Brasil & 1.067 .962 & 0,452 \\
México & 839.182 & 0,356 \\
Argentina & 214.058 & 0,091 \\
Chile & 145.841 & 0,062 \\
Peru & 93.269 & 0,040 \\
\hline
\end{tabular}

Fonte: World Development Indicators Database, World Bank, 1 July 2007. Disponível em http://siteresources.worldbank.org/ DATASTATISTICS/Resources/GDP.pdf, acesso em 8 de dezembro de 2007. executado o teste $F$ sob a hipótese nula: $H_{0}: b_{1(\text { MERVAL })}=0,091$; $b_{2(I P S A)}=0,062 ; b_{3(I P C)}=0,356 ; b_{4(\text { Lima Gen. })}=0,040$.

A estatística $F$ resultante de 5,805 e p-valor de 0,001 rejeitam a hipótese nula, ou seja, o portfólio de risco mínimo é estatisticamente diferente de uma carteira com pesos proporcionais ao PIB de cada país.

De forma a testar o portfólio de tangência a partir da taxa livre de risco é construída a matriz de teste $\mathrm{R}$ como em Britten-Jones (1999). A hipótese nula neste caso pode ser enunciada como: $\left.H_{0}: b_{1(I B r X)} /\left(1^{\prime} * b\right)=0,452 ; \ldots ; b_{4(I P C)}\right)^{\prime}$ $(1 * b)=0,356$.

A estatística $F$ de 2,148 e p-valor de 0,095 não rejeitam a hipótese nula de que os pesos no portfólio de tangência são proporcionais ao PIB de cada país para o período analisado.

\section{Conclusão}

O problema de como alocar ativos eficientemente tem sido uma das questões em constante evolução em Finanças e, recentemente, alguns trabalhos têm se voltado para a inferência estatística sobre os resultados da fronteira eficiente.

Um tema que tem merecido atenção tanto por sua importância acadêmica quanto prática é o problema de diversificação pela inclusão de outros países na carteira, em adição ao mercado doméstico.

Este trabalho propôs utilizar a combinação das metodologias de inferência por regressão desenvolvidas por Britten-Jones (1999), Kempf e Memmel (2006) para inferência estatística sobre pesos na fronteira eficiente, e estendeu sua análise ao caso do investidor brasileiro. Foi analisada a possibilidade de diversificação pela aplicação em demais países da América Latina.

Os resultados permitiram verificar que, embora os pesos nos demais países no portfólio de tangência não sejam diferentes de zero ao nível de significância de 5\%, alguns dos pesos são estatisticamente significantes no caso do portfólio de risco mínimo global, também ao nível de 5\% de significância. Portanto, a adição de outros mercados da América Latina melhoraria a fronteira eficiente em relação à aplicação única em Brasil, sob o ponto de vista do investidor local. No caso em que não são incluídas restrições à venda a descoberto o teste é similar a um teste de spanning.

Foi ainda verificada se uma carteira com pesos proporcionais ao PIB de cada país poderia ser considerada eficiente. A eficiência deste portfólio foi rejeitada para a carteira de risco mínimo, mas não foi rejeitada para o portfólio de tangência tomado a partir da caderneta de poupança como ativo livre de risco.

Trabalhos adicionais poderiam ampliar o universo de países analisados ou executar a análise para outras classes de ativos que não apenas ações. 
BRITTEN-JONES, M. The sampling error in estimates of meanvariance efficient portfolio weights. The Journal of Finance, v. LIV, n. 2, p. 655-671, 1999.

BRUNI, A. L.; FUENTES, J.; FAMÁ, R. A moderna teoria de portfólios e a contribuição dos mercados latinos na otimização da relação risco versus retorno de carteiras internacionais: evidências empíricas recentes (1996-1997). In: SEMINÁRIOS EM ADMINISTRAÇÃO, 3., 1998, São Paulo. Anais... São Paulo: FEA - USP, 1998. Disponível em http://www.ead.fea.usp.br/ Semead $/ 3$ semead/Financas.html. Acesso em 10 outubro 2006.

CAMPBELL, J. Y.; LO, A. W.; MacKINLAY, C. A. The econometrics of financial markets. New Jersey: Princeton University Press, 1997. $611 \mathrm{p}$.

CHOPRA, V. K.; ZIEMBA, W. T. The effect of errors in means, variances, and covariances on optimal portfolio choice. The Journal of Portfolio Management, Winter, p. 6-11, 1993.

DEROON, F. A.; NIJMAN, T. E.; WERKER, B. J. M. Testing for mean-variance spanning with short sales constraints and transaction costs: the case of emerging markets. The Journal of Finance, v. LVI, n. 2, p. 721-742, 2001.

DOORNIK, J. A.; HANSEN, H. An omnibus test for univariate and multivariate normality: working paper. 16 p. Disponível em: $<$ http://citeseer.ist.psu.edu>. Acesso em: 08 de Outubro de 2006.

ELTON, E. J. et al. Moderna teoria de carteiras e análise de investimentos. São Paulo: Atlas, 2004. 602 p.

FAMÁ, R.; PEREIRA, L. M. Diversificação internacional de portfólios e a integração dos mercados em desenvolvimento na América Latina e Estados Unidos. In: SEMINÁRIOS EM ADMINISTRAÇÃO, 6., 2003, São Paulo. Anais... São Paulo: FEA - USP, 2003. Disponível em http://www.ead.fea.usp.br/ Semead/6semead/index.html. Acesso em 10 outubro 2006.

FLETCHER, J.; MARSHALL, A. An empirical examination of the benefits of international diversification. Journal of International Financial Markets, Institutions \& Money, v. 15, n. 5, p. 455-468, 2005.

GUJARATI, D. N. Econometria básica. 3 ed. São Paulo: Pearson Makron Books, 2000. 846 p.

HUBERMAN, G.; KANDEL, S. Mean-variance spanning. The Journal of Finance, v. XLII, n. 4, p. 873-888, 1987.

JORION, P. Portfolio optimization in practice. Financial Analysts Journal, v. 48, n. 1, p. 68-74, 1992.
KAN, R.; ZHOU, G. Tests of mean-variance spanning. 2001. Working Paper. Disponível em <http://ssrn.com/abstract=231522>. Acesso em: 10 de Outubro de 2006.

KEMPF, A.; MEMMEL, C. Estimating the global minimum variance portfolio. Schmalenbach Business Review, v. 58, p. 332-348, 2006.

KROLL, Y.; LEVY, H.; MARKOWITZ, H. M. Mean-variance versus direct utility maximization. The Journal of Finance, v. XXXIX, n. 1, p. 47-61, 1984.

LI, K.; SARKAR, A.; WANG, Z. Diversification benefits of emerging markets subject to portfolio constraints. Journal of Empirical Finance, v. 10, n. 1-2, p. 57-80, 2003.

MARKOWITZ, H. Portfolio Selection. The Journal of Finance, v. VII, n. 1, p. 77-91, 1952.

MATILLA-GARCIA, M. A non-parametric test for independence based on symbolic dynamics. Journal of Economic Dynamics \& Control, v. 31, n. 12, p. 3889-3903, 2007.

MICHAUD, R. O. The Markowitz optimization enigma: is 'optimized' optimal? Financial Analysts Journal, v. 45, n. 1, p. 31-42, 1989. Efficient asset management. Boston: Harvard Business School Press, 1998. 152 p.

OLIVEIRA, M. A. C.; SILVA, L. S. A. Incerteza nos pesos na fronteira eficiente e a inclusão de ações da América latina sob o ponto de vista do investidor brasileiro. In: SIMPÓSIO DE ENGENHARIA DE PRODUÇÃO, 14., 2007, Bauru. Anais... Bauru: Universidade Estadual Paulista "Júlio de Mesquita Filho", 2007.

R DEVELOPMENT CORE TEAM. R: a language and environment for statistical computing. Vienna: R Foundation for Statistical Computing, 2008. Disponível em: <http://www.R-project.org>.

SOLNIK, B. Why not diversify internationally rather than domestically? Financial Analysts Journal, v. 30, n. 4, p. 48-54, 1974.

International investments. 3 ed. Massachusetts: AddisonWesley Publishing Co, 1996. 635 p.

TRAPLETTI, A.; HORNIK, K. Tseries: time series analysis and computational finance. R package version 0.10-16, 2008.

WICKHAM, P. Normwn.test: normality and white noise testing. R package version 1.0, 2008.

ZEILEIS, A.; HOTHORN, T. H. Lmtest diagnostic checking in regression relationships. $\mathrm{R}$ package, 2002.

\section{The inclusion of Latin American stocks for brazilian investors: inferences about efficient portfolio weights}

\section{Abstract}

Allocating resources efficiently has been one of the major issues in Finance. If domestic factors are the key reasons for local assets to move together, the investor should search for other markets in order to diversify the local risk. This topic has been analyzed considering the risk-return tradeoff. However, one of the main problems is not taking the uncertainty input parameters into account triggering estimation risk concerns. This article analyzes whether the inclusion of stocks from other Latin American countries improve the efficient frontier from a Brazilian investor's point of view. The combination of inferences about tangency portfolio (BRITTEN-JONES, 1999) and global minimum risk portfolio (KEMPF e MEMMEL, 2006) was implemented. From the results, it can be concluded that the inclusion of other Latin American stocks would improve the efficient frontier for local investors with statistically significant portfolio weights.

Keywords: Portfolio. Diversification. Inference. 


\section{Sobre os autores}

\section{Marco Antonio Cunha de Oliveira, CFA}

Universidade Federal do Rio de Janeiro - UFRJ

Rua Comendador Queiroz, 38, apto.1401, Icaraí, Niterói - RJ, CEP 24230-220

e-mail: Ismao@urbi.com.br

\section{Lílian Simone Aguiar da Silva}

Rua Domingues de Sá, 230, apto. 503, Icaraí, Niterói - RJ, CEP 24220-091

e-mail: Isas@ urbi.com.br

Recebido: 19/2/2009

Aceito: $27 / 4 / 2009$ 